\title{
Cap-independent translation by DAP5 controls cell fate decisions in human embryonic stem cells
}

\author{
Yael Yoffe, ${ }^{1}$ Maya David, ${ }^{1}$ Rinat Kalaora, ${ }^{1}$ Lital Povodovski, ${ }^{1}$ Gilgi Friedlander, ${ }^{2}$ Ester Feldmesser, ${ }^{3}$ \\ Elena Ainbinder, ${ }^{4}$ Ann Saada, ${ }^{5,6}$ Shani Bialik, ${ }^{1}$ and Adi Kimchi ${ }^{1}$ \\ ${ }^{1}$ Department of Molecular Genetics, ${ }^{2}$ Nancy and Stephen Grand Israel National Center for Personalized Medicine, ${ }^{3}$ Bioinformatics \\ Unit, ${ }^{4}$ Stem Cell Core Unit, Weizmann Institute of Science, Rehovot 76100, Israel; ${ }^{5}$ Monique and Jacques Roboh Department of \\ Genetic Research, ${ }^{6}$ Department of Genetics and Metabolic Diseases, Hadassah-Hebrew University Medical Center, Jerusalem \\ 91120, Israel
}

\begin{abstract}
Multiple transcriptional and epigenetic changes drive differentiation of embryonic stem cells (ESCs). This study unveils an additional level of gene expression regulation involving noncanonical, cap-independent translation of a select group of mRNAs. This is driven by death-associated protein 5 (DAP5/eIF4G2/NAT1), a translation initiation factor mediating IRES-dependent translation. We found that the DAP5 knockdown from human ESCs (hESCs) resulted in persistence of pluripotent gene expression, delayed induction of differentiation-associated genes in different cell lineages, and defective embryoid body formation. The latter involved improper cellular organization, lack of cavitation, and enhanced mislocalized apoptosis. RNA sequencing of polysome-associated mRNAs identified candidates with reduced translation efficiency in DAP5-depleted hESCs. These were enriched in mitochondrial proteins involved in oxidative respiration, a pathway essential for differentiation, the significance of which was confirmed by the aberrant mitochondrial morphology and decreased oxidative respiratory activity in DAP5 knockdown cells. Further analysis identified the chromatin modifier HMGN3 as a cap-independent DAP5 translation target whose knockdown resulted in defective differentiation. Thus, DAP5-mediated translation of a specific set of proteins is critical for the transition from pluripotency to differentiation, highlighting the importance of capindependent translation in stem cell fate decisions.
\end{abstract}

[Keywords: DAP5; cap-independent translation; human embryonic stem cells; stem cell differentiation; protein translation control]

Supplemental material is available for this article.

Received June 9, 2016; revised version accepted August 18, 2016.

Death-associated protein 5 (DAP5/eIF4G2/NAT1) is a member of the eIF4G translation initiation factors, which assemble on the mRNA's 5' cap structure as the preinitiation complex (Imataka et al. 1997; Levy-Strumpf et al. 1997; Shaughnessy et al. 1997; Yamanaka et al. 1997). eIF4G is a scaffold for binding eIF4E, the cap recognition factor; eIF4A, the RNA helicase; eIF3, which recruits the 40S ribosome; and poly(A)-binding protein (PABP). DAP5, however, lacks eIF4G's $\mathrm{N}$ terminus, including the eIF4E- and PABP-interacting domains, and is thus incapable of mediating cap-dependent translation (Imataka et al. 1997; Levy-Strumpf et al. 1997; Yamanaka et al. 1997; Liberman et al. 2015). Rather, it mediates cap-independent translation through a mechanism involving the recruitment of the ribosome directly to specific mRNAs

Corresponding author: adi.kimchi@weizmann.ac.il

Article is online at http://www.genesdev.org/cgi/doi/10.1101/gad.285239. 116. Freely available online through the Genes \& Development Open Access option. that contain an internal ribosome entry site (IRES) in their 5' untranslated region (UTR). DAP5 stimulates IRES-dependent translation of cellular mRNAs in rabbit reticulocyte lysates (RRLs) but is completely dispensable for capdependent translation (Liberman et al. 2015). In cells, DAP5 supports IRES-driven translation of c-Myc, Apaf1, c-IAP2, and p53 under apoptotic or stress conditions (Henis-Korenblit et al. 2002; Nevins et al. 2003; Warnakulasuriyarachchi et al. 2004; Lewis et al. 2008; WeingartenGabbay et al. 2014) and, in nonstressed cells, drives IRESmediated translation of Bcl-2 and CDK1 during the mitotic phase of the cell cycle (Marash et al. 2008). DAP5 itself contains an IRES in its $5^{\prime}$ UTR, thus creating a positive autoregulatory loop enabling its continuous translation under conditions in which cap-dependent translation is compromised (Henis-Korenblit et al. 2000; Lewis et al.

(C) 2016 Yoffe et al. This article, published in Genes \& Development, is available under a Creative Commons License (Attribution-NonCommercial 4.0 International), as described at http://creativecommons.org/licenses/by-nc/4.0/. 
2008). Notably, a recent study showed that DAP5 also participates in the translation of specific mRNAs in quiescent cells and immature oocytes through interactions with an FXR1a-miRNP complex that binds the 3' UTR (Bukhari et al. 2016).

DAP5 knockout mice die at an early stage of gastrulation (Yamanaka et al. 2000), and, similarly, knockdown of the zebrafish DAP5 ortholog leads to impaired mesoderm formation and early embryonic lethality (Nousch et al. 2007). In addition, DAP5 ${ }^{-/-}$mouse embryonic stem cells (mESCs) showed defective differentiation in response to retinoic acid (RA) or when grown as teratomas (Yamanaka et al. 2000). The molecular mechanisms driving this critical effect of DAP5 on early embryogenesis and differentiation and whether they result from DAP5's function as a translation factor have not yet been explored.

ESCs are pluripotent cells derived from the inner cell mass of blastocyst stage embryos (Thomson et al. 1998). They are maintained in a stable, self-renewing state and can differentiate into all cell types. Self-renewal and pluripotency are maintained by a complex regulatory network controlled mainly by master transcription factors, which in turn regulate other transcription factors, epigenetic modifiers, and signal transduction pathways. Differentiation and lineage specification involve major changes in gene expression, and significant progress has been made in understanding the transcriptional and epigenetic factors regulating these processes (for review, see Orkin and Hochedlinger 2011; Young 2011; Yeo and Ng 2013). Less is known about regulatory processes operating at the protein translation level, although initial studies have suggested that there is a global increase in protein translation and expression levels in differentiated cells and embryoid bodies (EBs) (Sampath et al. 2008; Novak et al. 2012). Conversely, undifferentiated human ESCs (hESCs) have lower levels of protein synthesis (Easley et al. 2010) and reduced activity of mTORC1, which may be responsible for reduced cap-dependent translation at this stage (Sampath et al. 2008; Easley et al. 2010; Tahmasebi et al. 2014). We hypothesized that, in pluripotent cells, under these conditions of parsimonious translation, noncanonical translation mechanisms may selectively increase the synthesis of particular proteins essential for facilitating the transition toward differentiation. We tested this hypothesis by examining DAP5's role as a cap-independent translation factor in hESC differentiation. We found that knockdown of DAP5 impaired differentiation in multiple differentiation models, concomitant with a reduced capacity to drive cap-independent translation. Polysomal RNA sequencing (RNA-seq) of DAP5 knockdown hESCs yielded a set of potential DAP5 target mRNAs with reduced translational efficiency, including a group of mitochondrial oxidative phosphorylation proteins and the nucleosome-binding protein HMGN3. HMGN3 was validated as a direct target of DAP5-mediated cap-independent translation whose knockdown partially phenocopied DAP5 knockdown. We suggest that selective translation of proteins through a cap-independent mechanism represents a new level of regulation of gene expression controlling the transition from pluripotency to differentiation.

\section{Results \\ DAP5-depleted hESCs fail to differentiate in response to multiple stimuli}

To study the functional role of DAP5 in ESC differentiation, we generated stable DAP5 knockdown cells by lentivirus infection of $\mathrm{H} 9$ hESCs with DAP5 or control nontargeting (NT) shRNA. When grown under undifferentiated conditions, DAP5 knockdown cells did not differ from controls in morphology, overall growth kinetics, karyotype, proliferation rates, or cell cycle distribution (Fig. 1A; Supplemental Fig. S1A-C). mRNA expression levels of pluripotent genes did not significantly differ between NT and DAP5 knockdown cells, with the exception of GDF3, whose mRNA levels increased (Fig. 1B). Similarly, immunostaining for pluripotent markers Nanog, Oct4, and Rex-1 indicated similar intensity and distribution over the whole colony (Fig. 1C; Supplemental Fig. S1D).

hESCs undergo a directed differentiation program to neural progenitor cells in response to RA, upon which pluripotent gene expression is suppressed concomitantly with the induction of differentiation-associated genes (Dhara and Stice 2008). RA treatment did not affect DAP5 protein expression in H9 cells or cells expressing either NT shRNA or NT siRNA (Fig. 1E; Supplemental Fig. S2A,B). Notably, DAP5 knockdown cells showed a significantly more moderate reduction in NANOG mRNA levels compared with NT hESCs upon treatment with RA (Fig. 1D). This was confirmed at the protein level by Western blotting for Nanog and Oct4, a second pluripotent marker whose protein expression declines more gradually following RA treatment (Fig. 1E). In parallel, the RA-induced increase in early differentiation-associated gene expression (namely, HOXA1 and $R A R B$ ) was compromised by the knockdown of DAP5 (Fig. 1D). Similar results were observed upon transient transfection of $\mathrm{H} 9$ cells with siRNA to DAP5 (Supplemental Fig. S2B). Importantly, reintroduction of exogenous DAP5 to the stable shRNA-expressing cells or DAP5 siRNA transfected cells increased HOXA1 and RARB mRNA expression to levels similar to those of the NT control cells (Fig. 1F; Supplemental Fig. S2C). Reintroduction of DAP5 into DAP5 shRNA knockdown cells also partially restored the decline in Nanog protein levels, which was compromised upon DAP5 depletion (Fig. 1F, right panel). This indicates that hESCs require DAP5 for the reported changes in gene expression that occur in response to RA. Extending this finding to additional stimuli indicated that various differentiation markers were suppressed in DAP5 knockdown compared with NT hESCs in response to BMP4, which triggers hESC differentiation to mesodermal lineages (Supplemental Fig. S2D).

To further show that the effect of DAP5 depletion on differentiation can be generalized to other differentiation programs, hESCs were induced to form EBs. In NT knockdown EBs, as expected, Nanog and Oct4 mRNA and protein levels started to drop at $3 \mathrm{~d}$ of EB formation and were then turned off completely. In contrast, the decline was delayed in DAP5 knockdown EBs, and the expression of these markers persisted for up to 3 wk of EB formation 
A
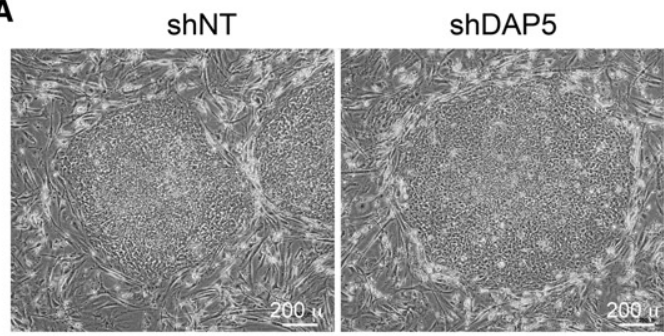

B

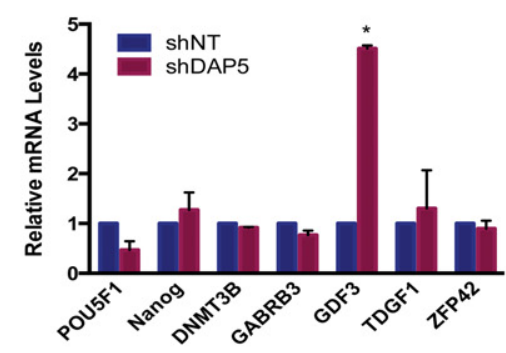

\section{C}
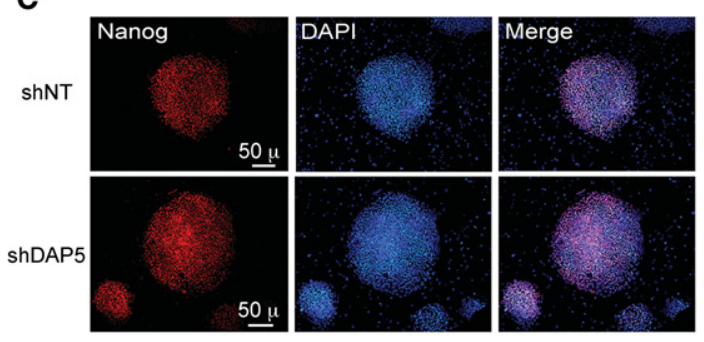
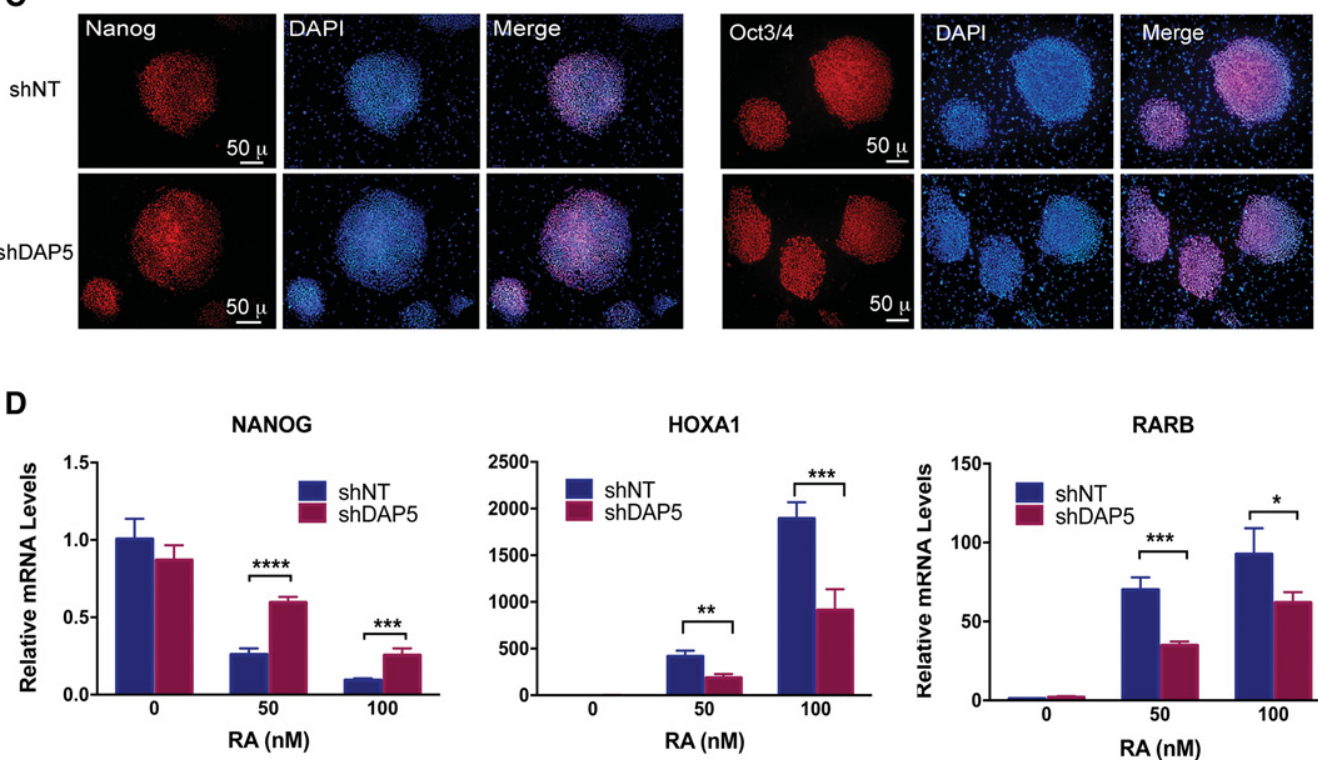

E

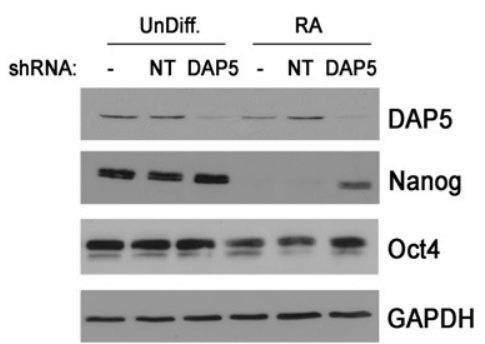

HOXA1

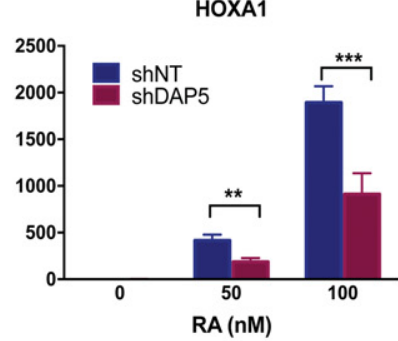

$\mathbf{F}$

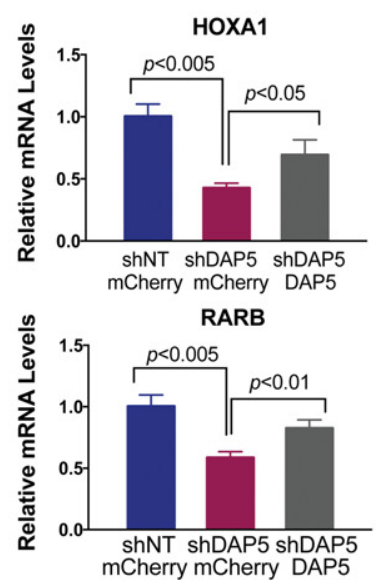

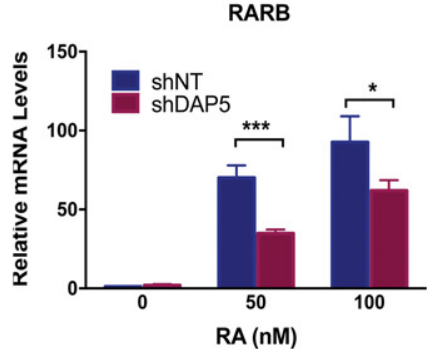

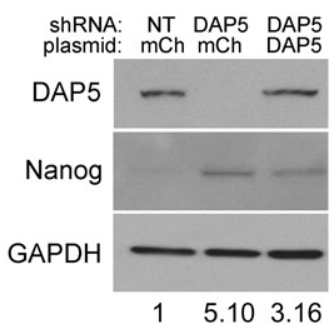

Figure 1. Phenotypic and molecular characterization of DAP5 knockdown hESCs. (A) Light microscopic images of NT and DAP5 shRNA-expressing hESCs grown on mouse embryonic fibroblast (MEF) feeder cells. (B) Quantitative real-time PCR was performed on nondifferentiated NT shRNA-expressing or DAP5 shRNA-expressing hESCs. For each gene, the mRNA level of the NT knockdown cells was set at 1 . Shown are means \pm SD of independent biological duplicates. Statistical significance between pairs was determined by $t$-test. $\left({ }^{*}\right) P<$ 0.005; others are not significantly different. $(C)$ NT and DAP5 knockdown hESC colonies were immunostained for plutipotent markers Nanog and Oct3/4. DAPI was used to stain nuclei. (D) Relative mRNA levels of NANOG, HOXA1, and RARB in NT or DAP5 knockdown hESCs treated with RA at the indicated concentrations for $\left.48 \mathrm{~h} .\left(^{*}\right) P<0.05 ;{ }^{* *}\right) P<0.01$; $\left(^{* * *}\right) P<0.005$; $\left(^{* * * *}\right) P<0.0005$. $(E)$ Western blot of wild-type H9 (-), control (NT), and DAP5 shRNA-expressing cells undifferentiated or treated with $100 \mathrm{nM}$ RA for 48 h, showing expression of DAP5 and pluripotent markers Nanog and Oct4. GAPDH was used as loading control. (F) NT and DAP5 shRNA hESCs were transfected with control mCherry or DAP5 plasmids and then treated with RA for 24 h. RT-PCR was performed to quantify mRNA levels of HOXA1 and RARB, and protein levels of Nanog were monitored by Western blotting. Densitometric analysis of Nanog protein levels, normalized to GAPDH protein levels, is presented below the blots. In the graphs in $D$ and $F$, data are presented as mean \pm SD of triplicates, with statistical significance determined by $t$-test. 
(Fig. 2A,B). Immunostaining for Nanog confirmed that it was expressed throughout the DAP5 knockdown EBs, as opposed to the reduced staining in control EBs (Fig. 2C). Similar effects on TRA-1-60 protein levels were observed upon FACS analysis (Supplemental Fig. 3A). A more comprehensive assessment of pluripotent markers using gene arrays in DAP5 and NT knockdown EBs revealed that most retained abnormally high levels of mRNA expression in DAP5 knockdown day 10 EBs compared with undifferentiated cells (Fig. 2D). The same results were obtained upon knockdown of DAP5 in the H1 hESC line (Supplemental Fig. S3B). Altogether, the failure to suppress pluripotent genes appears to be a robust outcome of loss of DAP5 expression.

Histological examination of EB sections indicated that DAP5 knockdown cells failed to form the pseudostratified columnar epithelial layer and the surrounding basement membrane, as revealed by $\mathrm{H} \& \mathrm{E}$ (Fig. 3A) and laminin (Supplemental Fig. S4A) staining. In addition, no organized cavitation was observed in DAP5 knockdown EBs, unlike NT and parental H9 cells EBs (Fig. 3A, day 14; Supplemental Fig. S4B). Surprisingly, numerous cells with apoptotic morphology were observed in DAP5-depleted EBs upon DAPI staining of nuclei (Fig. 3B), which was confirmed by staining for active caspase3 (Fig. 3C). In control EBs, fewer apoptotic cells were observed, and these were restricted to the cavity, as de- scribed previously (Coucouvanis and Martin 1995). In contrast, in DAP5 knockdown EBs, apoptotic cells were detected throughout the EB, up to its periphery (Fig. 3B, C). Furthermore, caspase-3-positive cells were TRA-160-positive (i.e. undifferentiated cells), whereas, in NT knockdown EBs, caspase-3-positive cells were mostly TRA-1-60-negative (Supplemental Fig. S4C). The same phenotype was observed in H1 DAP5 knockdown EBs (Supplemental Fig. S4D). Western blotting for active caspase- 3 and the cleavage of its substrate, ICAD, indicated earlier and stronger activation of apoptosis in the DAP5 knockdown EBs (starting at day 7 of EB formation compared with day 14 in the control EBs) (Fig. 3D). Interestingly, enhanced apoptosis was observed only in EBs; when compared with NT cells, DAP5 knockdown hESCs did not show any increase in caspase-3 activation upon differentiation by RA (Supplemental Fig. S4E). Thus, depletion of DAP5 only primes hESCs to apoptosis in the context of EB differentiation.

Addition of Q-VD, a peptide inhibitor of caspase-3 (Caserta et al. 2003), attenuated nuclear fragmentation in DAP5 knockdown EBs (Fig. 3E) and processing of caspase-3 on Western blots (Fig. 3F). However, while cavitation of H9 and NT knockdown cells was blocked by Q-VD (Fig. 3E; Supplemental Fig. S4F), the cellular disorganization and impaired differentiation morphology of the DAP5 knockdown EBs were not reversed (Fig. 3E).
A

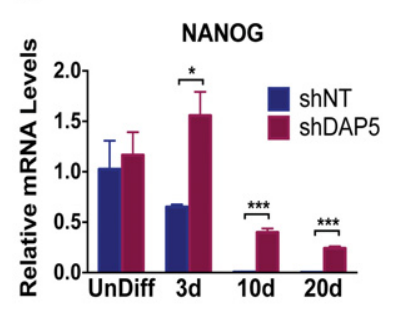

C

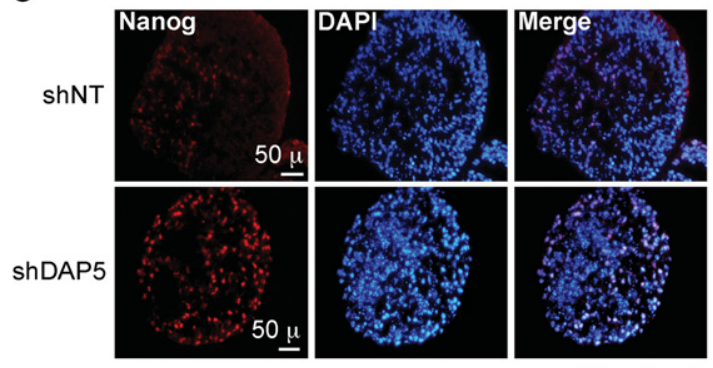

B

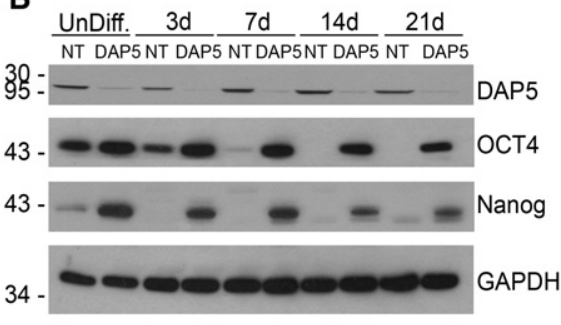

D

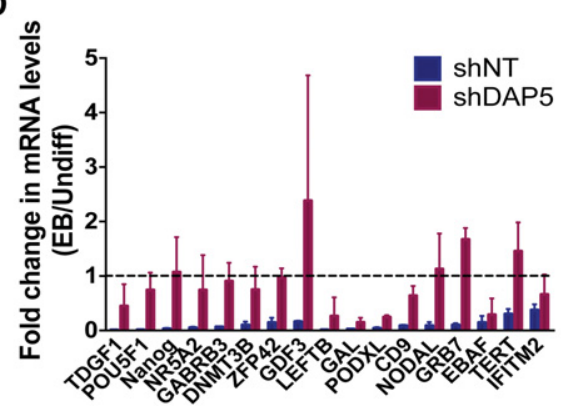

Figure 2. DAP5 depletion blocks the suppression of pluripotent protein expression in differentiating EBs. $(A)$ Relative mRNA levels of NANOG and OCT4 from NT or DAP5 knockdown cells in the undifferentiated state or from EBs grown for the indicated number of days. Data are presented as mean \pm SD of triplicates. Statistical significance was determined by $t$-test. $\left(^{*}\right) P<0.05 ;\left({ }^{* *}\right) P<0.01 ;\left(^{* * *}\right) P<0.005$. Note that the increase in NANOG and OCT4 expression in DAP5 knockdown on day 3 compared with undifferentiated is not significant. $(B)$ Western blot of NT and DAP5 knockdown hESCs comparing the expression levels of Oct 4 and Nanog in undifferentiated cells and on days $3,7,14$, and 21 of EB formation. GAPDH was used as loading control. (Due to the size range being similar to that of Oct4, the same lysates were run on a separate parallel gel and blotted for Nanog.) (C) Cryosections of 7-d DAP5 and NT knockdown EBs stained for Nanog and DAPI to visualize nuclei. $(D)$ Quantitative real-time PCR was performed on 10-d NT or DAP5 shRNA-expressing EBs in biological duplicates. Shown are mRNA levels of various pluripotent markers, expressed as the fold difference $( \pm S D)$ between EBs and undifferentiated hESCs for each gene. 
A

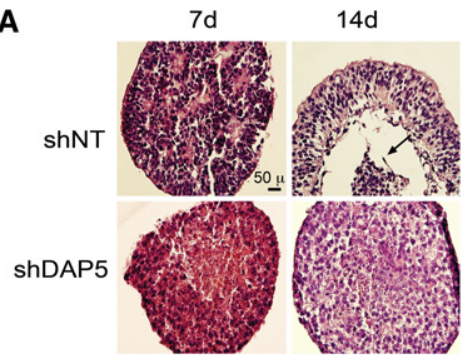

C

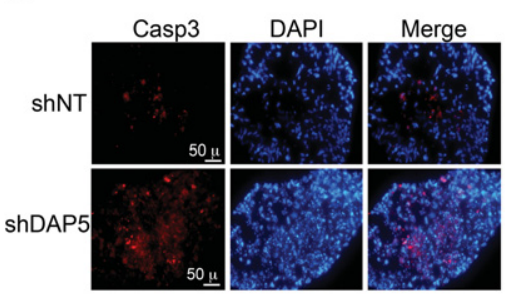

E

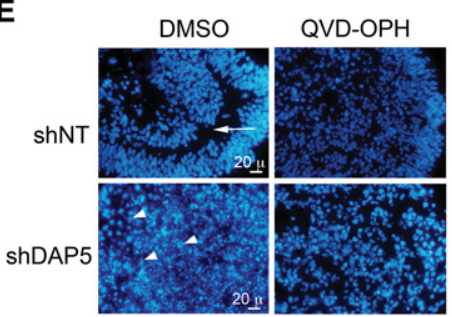

B

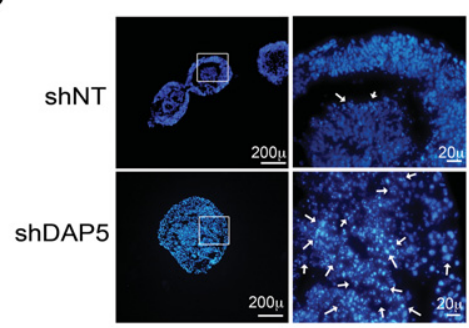

D

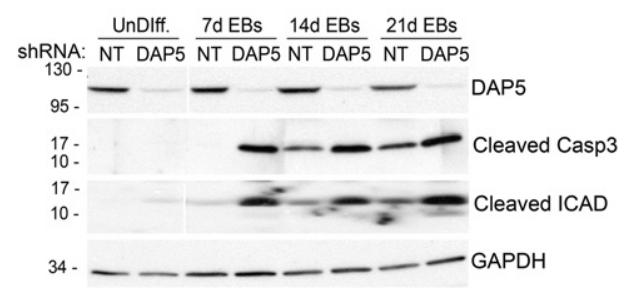

$\mathbf{F}$

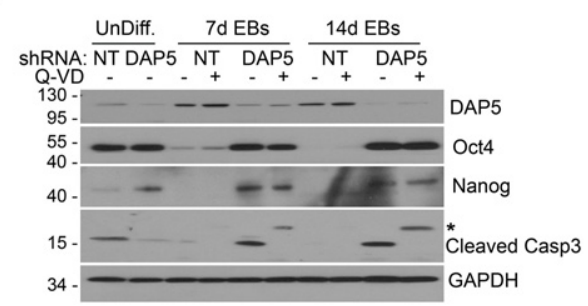

Figure 3. DAP5 knockdown EBs fail to differentiate and show enhanced apoptosis. (A) NT and DAP5 shRNA-expressing EBs were stained with H\&E at the indicated time points. Note the presence of a cavity (arrow) in control EBs but not DAP5 knockdown EBs. (B) Fourteen-day NT and DAP5 knockdown EBs were stained with DAPI. The right panels are magnifications of the boxed area in the left panels. Arrows indicate examples of fragmented nuclei. $(C)$ Seven-day NT or DAP5 shRNA-expressing EBs were stained for active casapse-3 and DAPI. $(D)$ Western blot of NT or DAP5 knockdown EBs for apoptotic markers active casapse-3 and ICAD. (DAP5 and ICAD were run on separate gels; lanes showing $3 \mathrm{~d}$ were removed for consistency with the other proteins analyzed. The lysates were the same as used in Fig. $2 \mathrm{~B}$, and the same DAP5 blot is shown.) GAPDH served as a loading control. (E) DAPI staining of 14-d NT and DAP5 knockdown EBs treated with DMSO or Q-VD-OPH. Arrow indicates cavitation, and arrowheads indicate examples of fragmented nuclei. $(F)$ Western blot of DAP5 and NT knockdown EBs treated with DMSO or Q-VD on day 7 or 14. The asterisk indicates an immature processing intermediate of caspase-3.

Consistent with this, Oct4 and Nanog protein levels remained abnormally high in DAP5 EBs treated with Q-VD at $7 \mathrm{~d}$ and $14 \mathrm{~d}$ (Fig. 3F). Thus, while the caspase inhibitor blocked the cell death response to DAP5 depletion, it did not rescue the failure to silence pluripotent gene expression, indicating that excessive cell death was not responsible for impaired differentiation.

\section{RNA-seq of polysomal fractions to identify mRNAs} with reduced translation efficiency (TE) in DAP5 knockdown cells

To assess the established function of DAP5 as a mediator of IRES-dependent translation in DAP5 knockdown cells, NT and DAP5 knockdown hESCs were transfected with an IRES reporter construct consisting of the firefly luciferase (FL) gene and a hairpin loop at the $5^{\prime}$ to prevent readthrough from cap-dependent translation (Fig. 4A, scheme). The DAP5 IRES (Weingarten-Gabbay et al. 2016) was used, since DAP5 is expressed in pluripotent hESCs and is known to regulate its own IRES-dependent translation (Henis-Korenblit et al. 2002; Marash et al. 2008). An empty hairpin-FL construct lacking an IRES was used as a negative control. As expected, significant luciferase activity was measured in NT cells expressing the DAP5 IRES-FL construct but not the empty construct. Importantly, luciferase activity was greatly reduced in the DAP5 knockdown hESCs (Fig. 4A), suggesting that DAP5-mediated cap-independent translation activity is compromised in pluripotent DAP5 knockdown cells.

The challenge was then to identify the subset of mRNAs whose TE is reduced in DAP5 knockdown hESCs, which may be responsible for the strong phenotype described above. Since the deficiency was observed in response to different differentiation triggers, it was hypothesized that DAP5 "primes" hESCs toward various lineages; that is, it may promote the translation of certain proteins in hESCs whose functions become indispensable when various differentiation triggers are applied. According to this hypothesis, DAP5's mRNA translation targets are predicted to be (1) critical for the transition from pluripotency to differentiation, and (2) already present in 
A

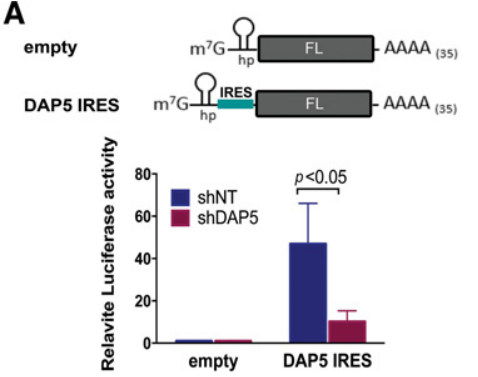

C

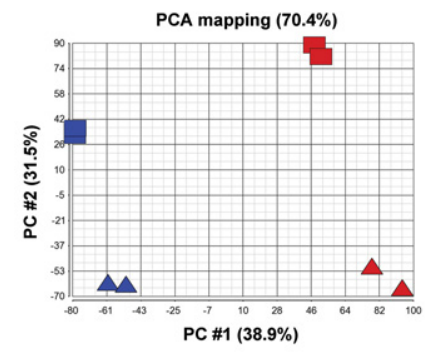

D

OxPhos/Mitochondria Annotation Cluster (enrichment score $=7.45$ )

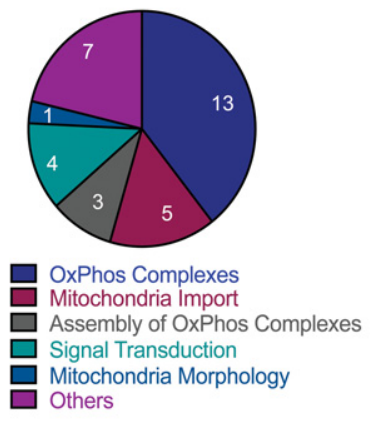

B

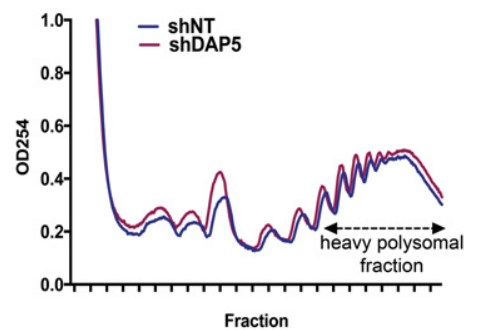

E shNT poly RNA - shNT total RNA

$\triangle$ ShDAP5 poly RNA

$\triangle$ shDAP5 total RNA

Ribosome/Translation Annotation Cluster (enrichment score $=7.09$ )

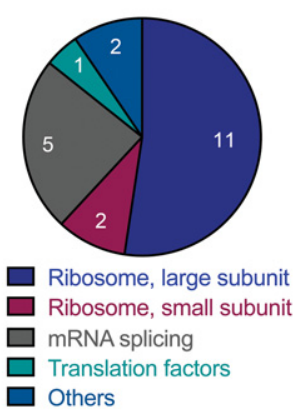

Figure 4. Identifying DAP5 translation targets by RNAseq of polysomal fractions. (A) Assessing IRES-dependent translation upon DAP5 knockdown. Luciferase activity was measured after transfection of NT or DAP5 knockdown cells with the constructs shown in the scheme above. (hp) Hairpin. Data are presented as mean \pm SD of triplicates. (B) Extracts from shNT or shDAP5 knockdown pluripotent $\mathrm{H} 9$ cells were loaded onto sucrose gradients, and $\mathrm{OD}_{254}$, representing RNA, was recorded. Fractions 19-23 represent heavy polysomes. (C) Analysis of deep RNA-seq results of total and polysomal-associated RNA in DAP5 and NT knockdown hESCs. Principal component analysis (PCA) of transcript profiles showing the variability between the samples (closer samples are more similar). The three axes account for $70.4 \%$ of the total variance among the samples. The PCA was calculated using a correlation matrix. (D) Annotation of the 122 potential DAP5 targets. The two gene ontology annotation clusters with the highest enrichment scores as calculated by DAVID were manually subcategorized into individual pie charts, with the number of proteins in each specific subcategory indicated. pluripotent hESCs. We therefore undertook an unbiased genome-wide polysomal screen to identify mRNA targets of DAP5 whose TE is reduced in pluripotent DAP5 knockdown hESCs.

To this end, mRNA from DAP5 and NT knockdown hESCs was subjected to polysomal fractionation to isolate heavy polysomes corresponding to the mRNA undergoing active translation. Analysis of DAP5-depleted and control cells showed no major differences in overall polysome profile, indicating that DAP5 knockdown does not have a major effect on global protein translation (Fig. 4B). mRNA from fractions corresponding to heavy polysomes were subjected to RNA-seq in biological duplicates to determine the relative abundances of actively translated mRNAs. Total mRNA was also sequenced to detect possible changes in gene expression upon DAP5 knockdown.

Data analysis indicated small variations between biological replicates, confirming reproducibility of the assays (Fig. 4C). In order to identify the mRNAs whose translation depends on DAP5, the TE was calculated for each gene by comparing the relative number of reads in the polysomal fractions versus total mRNA to exclude variations emerging from changes in mRNA abundance. The TE of shDAP5 samples was then compared with that of shNT samples to obtain the differential TE $(\Delta \mathrm{TE})$. Out of
13,200 detected genes, 122 genes had a $\Delta \mathrm{TE}$ showing a statistically significant reduction of at least 1.5 -fold (Supplemental Table S1). Gene ontology analysis by the DAVID algorithm (Huang et al. 2009a,b) indicated that nearly half of the genes were clustered in two major functional annotation groups: (1) mitochondrial proteins, especially components of the oxidative respiratory chain, and (2) proteins involved in translation, including many ribosomal proteins and mRNA splicing factors (Fig. 4D). In addition, several candidates had some previously known connection to embryogenesis and/or differentiation, such as HMGN3, ANAPC5, and ZDHHC4. To assess the reliability of the RNA-seq data, a small test group was subjected to quantitative real-time PCR, confirming a significant change ( $>30 \%$ reduction) in $\Delta \mathrm{TE}$ in 12 out of 16 genes (75\%) (Supplemental Table S1).

\section{Mitochondrial cluster}

Interestingly, the list of candidate mRNA targets with reduced TE emerging from the RNA-seq screen contained numerous nuclear-encoded genes functioning either within the oxidative phosphorylation pathway or as factors involved in their mitochondrial import and assembly (Fig. 4D). To determine whether oxidative respiration was 
affected in these cells, the activity of electron transport chain complex I was measured directly. In the pluripotent state, complex I activity was significantly lower in DAP5 knockdown cells compared with NT knockdowns (Fig. 5A). Mitochondria morphology also differed between NT and DAP5 knockdown hESCs undergoing RA-induced differentiation. In differentiated NT knockdown cells, mitochondria were elongated and filamentous, consistent with previous descriptions of mature mitochondria in differentiating hESCs (Mandal et al. 2011). In contrast, in DAP5 knockdown cells, mitochondria remained globular and clustered in the perinuclear area, resembling the morphology of the undifferentiated cells (Fig. 5B). These results confirm a reduction in mitochondrial function. To determine whether impaired mitochondrial oxidative respiratory activity could block RA-induced differentiation of hESCs, parental H9 cells were treated with rotenone, an inhibitor of complex I, simulating the defect observed in the DAP5 knockdown hESCs. Indeed, rotenone blocked RA-induced differentiation, as evidenced by the compromised induction in HOXA1 mRNA and maintenance of NANOG mRNA expression (Fig. 5C). Thus, hESCs fail to properly differentiate in response to RA when oxidative phosphorylation is impaired, underscoring the importance of an intact oxidative respiration pathway for hESC differentiation.
HMGN3 is a validated DAP5 target mRNA at both the translation and functional levels

In order to identify direct DAP5 targets among the candidates, several of the PCR-validated candidate target mRNAs were screened for cap-independent activity in an in vitro translation assay in RRLs (Liberman et al. 2015). The entire $5^{\prime}$ UTR of each mRNA was cloned into the hairpin-FL reporter construct (Fig. 6A). The BCL2 IRES was used as a positive control (Marash et al. 2008). Notably, the activity of the HMGN3 5' UTR was as high as the BCL2 IRES, suggesting that it can drive cap-independent translation (Fig. 6B). Next, the HGMN3 5' UTR and empty hairpin reporters were transfected into NT and DAP5 knockdown hESCs, and luciferase activity was monitored (Fig. 6C). The HMGN3 5' UTR resulted in increased translation of the luciferase reporter in the NT cells compared with the empty control. Significantly, this translation activity was reduced in the DAP5 knockdown cells, confirming that HMGN3 IRES activity is dependent on DAP5.

To determine whether DAP5 engages the HMGN3 mRNA, as would be expected of a direct translation target, protein-RNA immunoprecipitations were performed (Fig. 6D, scheme). hESCs were UV-cross-linked, and DAP5RNA complexes were immunoprecipitated with anti-
A

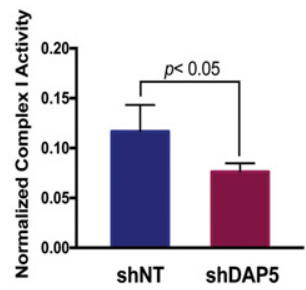

C

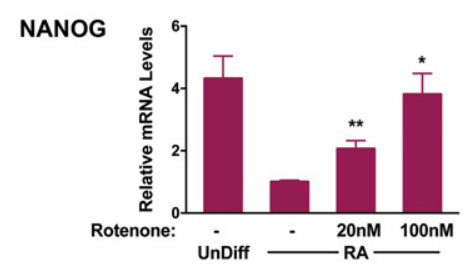

HOXA1

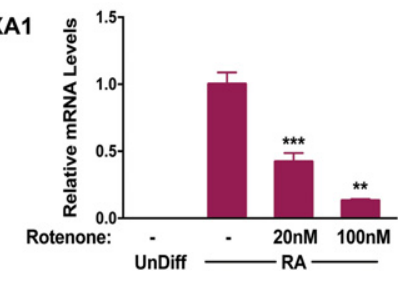

B

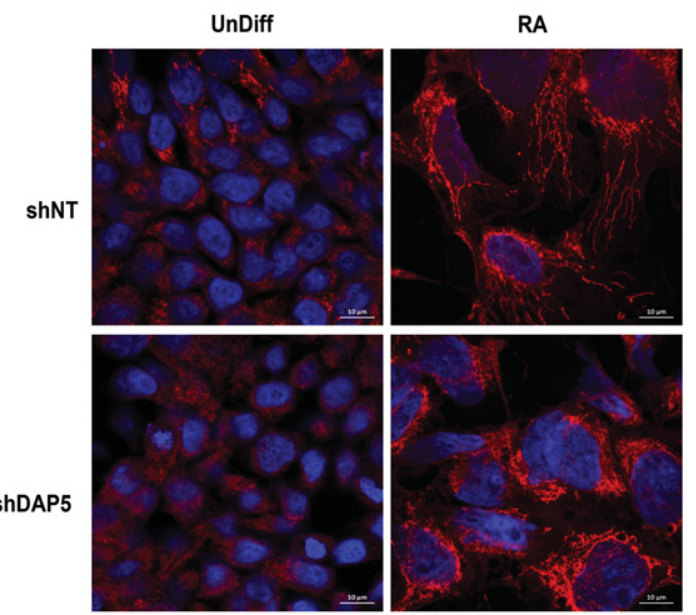

Figure 5. The effects of DAP5 knockdown on oxidative respiration. (A) Respiratory chain complex I activity in control and DAP5 knockdown hESCs was measured by spectrophotometry and normalized to citrate synthase activity, used as a control for mitochondria purification. Means \pm SD of normalized data from four experiments are shown. Significance was determined by $t$-test. $(B)$ DAP5 and NT knockdown hESCs were treated with $100 \mathrm{nM}$ RA for $24 \mathrm{~h}$ and stained with Mitotracker and DAPI. A higher exposure is shown for undifferentiated cells to visualize mitochondria morphology. $(C) \mathrm{H} 9$ cells were treated with $1 \mu \mathrm{M}$ RA for $24 \mathrm{~h}$ in the presence of increasing concentrations of rotenone, as indicated. Levels of NANOG and HOXA1 mRNA were measured by real-time PCR and graphed as mean \pm SD. Shown are representative graphs of three independent experiments. 
DAP5 antibodies. RNA purified from the DAP5 immunocomplexes was then subjected to quantitative PCR with primers to HMGN3, DAP5 as a positive control, and OCT4, whose TE does not change upon DAP5 depletion, as a negative control. HMGN3 mRNA was found in signif- icant levels in the DAP5 immunocomplex, indicating that it is bound to DAP5 in cells (Fig. 6D).

Since we showed previously that DAP5 can directly interact in vitro with specific IRES elements (WeingartenGabbay et al. 2014), to further characterize the DAP5-
A

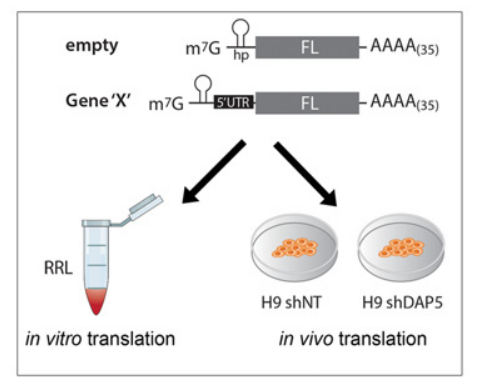

B

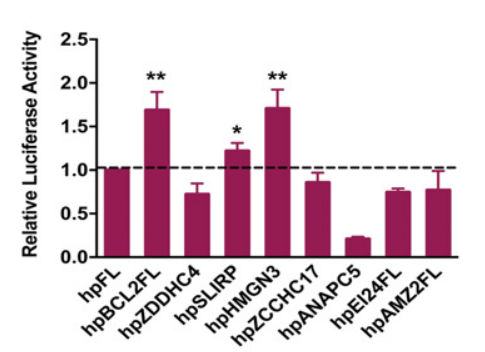

C

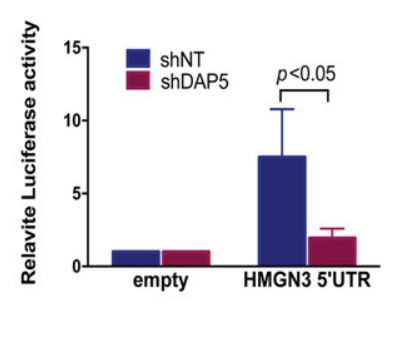

D
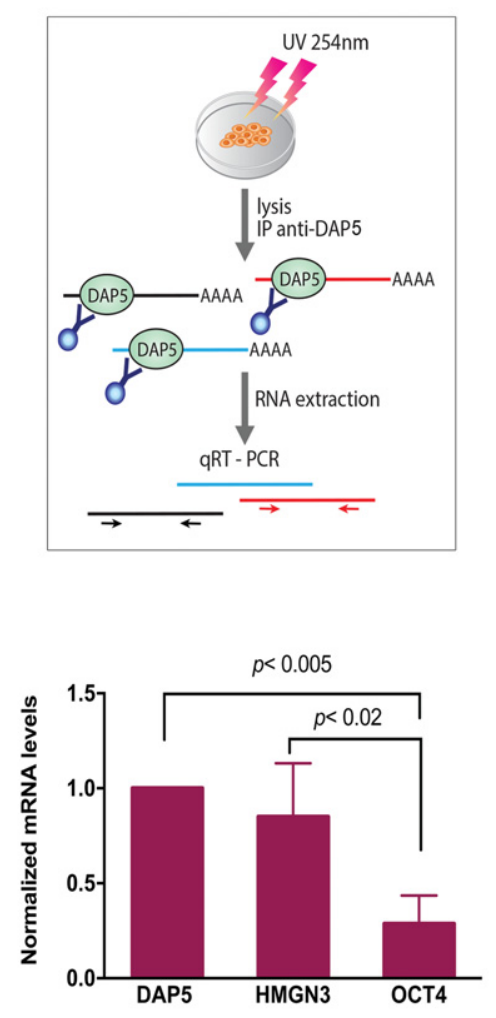

E
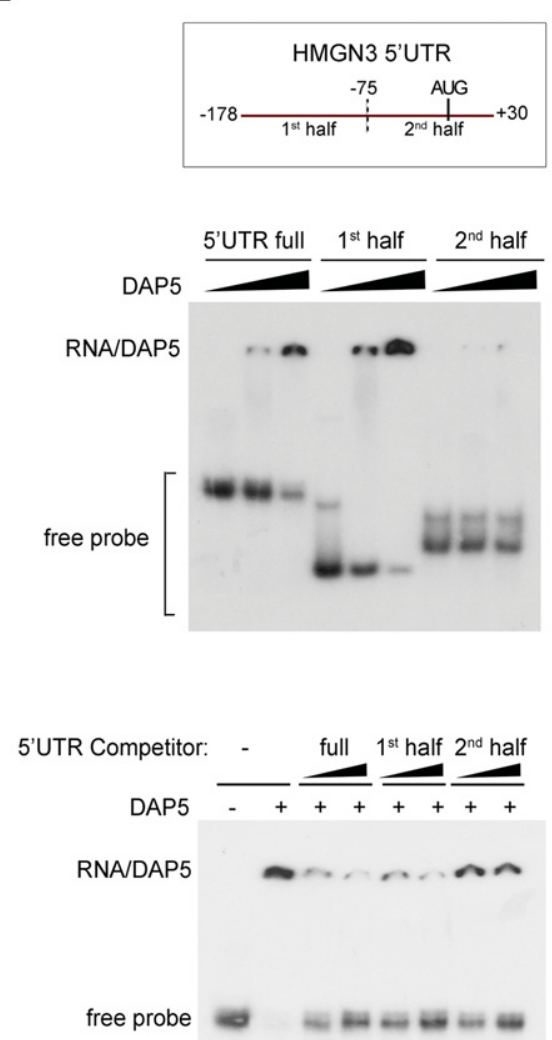

Figure 6. HMGN3 is a direct target of IRES-dependent DAP5-mediated translation. (A) Schematic of in vitro and in vivo IRES activity assays. (B) In vitro IRES activity screen. RNA hairpin (hp) FL vectors containing the $5^{\prime}$ UTRs of the indicated genes were translated in RRLs. Luciferase activity was measured and compared with background luciferase activity from the empty hairpin vector, which was set as $1 .\left(^{*}\right) P<0.02 ;(* *) P<0.005$. (C) IRES activity in vivo. HMGN3 $5^{\prime}$ UTR and empty hairpin vectors were transfected into NT and DAP5 knockdown hESCs, and relative luciferase activity was measured. $(D)$ DAP5 was immunoprecipitated from H9 pluripotent cells followed by purification of associated RNA. mRNA levels of the indicated genes were determined by real-time PCR and normalized to corresponding levels within total mRNA. Levels of DAP5 self-immunoprecipitation, used as a positive control, were set as 1 . (E, top) Radiolabeled RNAs corresponding to the HMGN3 5' UTR and the first or second half of the 5' UTR were incubated with or without DAP5 protein and resolved by gel electrophoresis. (Bottom) The radiolabeled HMGN3 5' UTR was incubated with DAP5 protein in the presence of increasing concentrations of competing unlabeled full-length $5^{\prime}$ UTR or either half. In all graphs, data are presented as mean \pm SD of three $(B, C)$ or four $(D)$ biological repeats. Significance was determined by $t$-test. 
dependency of the putative HMGN3 IRES, the region comprising HMGN3's 5' UTR was used in direct RNAbinding assays by EMSA (electophoretic mobility shift assay). A radioactive RNA probe corresponding to the region from -178 to +30 nucleotides (nt) past the start site was generated and incubated with purified recombinant DAP5 protein. Significant binding of the $5^{\prime}$ UTR to DAP5 was observed (Fig. 6E). The specificity of this interaction was confirmed by dividing the $5^{\prime}$ UTR into two equal fragments (Fig. 6E, scheme). Only the first half of the HMGN3 5' UTR interacted with DAP5 (Fig. 6E). Moreover, the interaction of DAP5 with the full 5' UTR was attenuated in a dose-dependent manner by addition of cold RNA corresponding to either the full $5^{\prime}$ UTR or the first half. Thus, DAP5 directly binds specific elements in the region of nucleotides -178 to -75 of the HMGN3 5' UTR. Altogether, these assays determined that HMGN3 is a direct translation target of DAP5, undergoing DAP5-mediated cap-independent translation through its $5^{\prime}$ UTR in hESCs.

HMGN3 is a chromatin-binding protein effecting epigenetic changes on gene expression (Ueda et al. 2009; Barkess et al. 2012). As such, it may function as a critical upstream mediator within a DAP5-initated hierarchy to control hESC differentiation. Therefore, the function of HMGN3 was examined in hESCs. HMGN3 protein was readily detected on Western blots in pluripotent hESCs (Fig. 7A). Protein expression levels were significantly reduced in DAP5 knockdown hESCs compared with controls, while mRNA levels were actually slightly elevated (Fig. 7A,B), validating the reduction in TE observed. HMGN3 was knocked down by siRNA in H9 hESCs (Fig. 7C), which were then triggered to undergo differentiation by administration of RA. The knockdown of HMGN3 attenuated the classical decline in NANOG mRNA levels seen in the control NT cells after 24 or $48 \mathrm{~h}$ of RA treatment, similar to the response of DAP5 knockdown cells (Fig. 7D). Nanog protein levels likewise remained higher in differentiated HMGN3 knockdown cells compared with NT knockdown cells (Fig. 7E). Also, the increase in differentiation marker HOXA1 mRNA levels seen in NT cells was delayed in siDAP5 and siHMGN3 transfected cells (Fig. 7D). Thus, HMGN3 knockdown at least partially phenocopies DAP5 knockdown and is necessary for hESC differentiation.

\section{Discussion}

In this study, we showed that the translation factor DAP5 is indispensable for differentiation of hESCs in response to multiple differentiation stimuli. Its knockdown resulted in persistence of pluripotent gene expression, delayed induction of differentiation-associated genes, and defective EB formation. The latter involved improper cellular organization, lack of cavitation, and aberrant apoptosis in terms of quantity, location, and onset. This could result from the defective differentiation of DAP5 knockdown EBs, for example, due to the absence of a basement membrane and its associated survival signals, which protect the outer ectoderm from death signals that activate apoptosis within the internal ectodermal layer, driving cavitation (Coucouvanis and Martin 1995, 1999). Alternatively, impaired differentiation and enhanced apoptosis may be two independent phenotypes of DAP5 depletion resulting from alterations in the expression of DAP5 target genes. For example, defective oxidative respiration may further sensitize DAP5 knockdown EBs to the hypoxic conditions within the structure, which has been shown previously to regulate apoptosis within the EB core by upregulating BNIP3 (Qi et al. 2012). In fact, RNA-seq of total mRNA indicated an increase in BNIP3 mRNA in DAP5 knockdown hESCs (data not shown). Notably, blocking

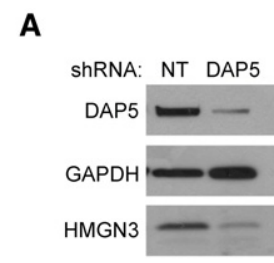

D

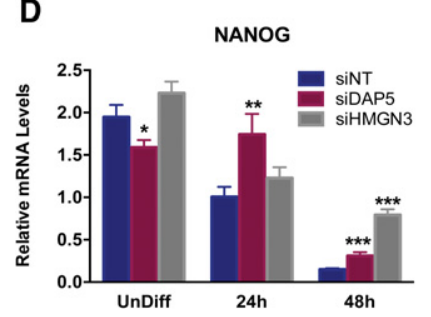

B
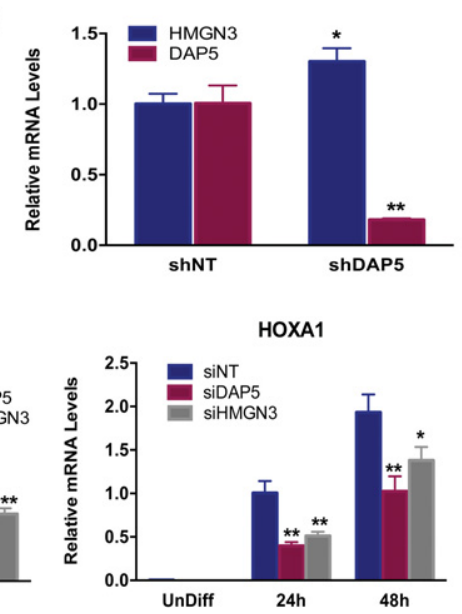

C

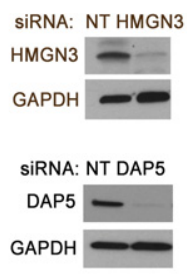

E

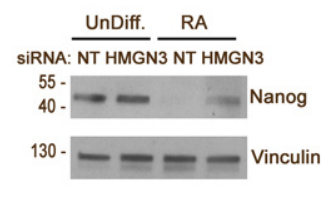

Figure 7. HMGN3 functions downstream from DAP5 and is necessary for differentiation. Protein $(A)$ or mRNA $(B)$ levels of DAP5 and HMGN3 in NT or DAP5 knockdown pluripotent cells. $\left(^{*}\right) P<0.05$; $\left.{ }^{* *}\right) P<0.01$. (C) HMGN3 or DAP5 was knocked down in H9 pluripotent cells using siRNA, as confirmed by Western blotting. (D) Relative mRNA expression levels of NANOG and HOXA1 in HMGN3 and DAP5 knockdown hESCs were measured after RA-induced differentiation. $\left(^{*}\right) P<0.05$; $\left(^{* *}\right) P<0.01$; $\left(^{* * *}\right) P<0.005$, knockdown versus NT. (E) Western blotting of NT and HMGN3 knockdown cells treated with RA to show Nanog levels. Vinculin was used as loading control. In all graphs, data are presented as mean \pm SD of triplicates. Statistical significance was determined by $t$-test. 
apoptosis with a caspase inhibitor did not rescue the differentiation defect, indicating that excessive cell death was not responsible for impaired differentiation.

The fact that DAP5 function was not limited to a specific differentiation pathway or cell lineage suggests that DAP5-depleted pluripotent hESCs are inherently less primed to differentiate compared with their control counterparts. Mechanistically, this can be attributed to the inability of DAP5 knockdown hESCs to support capindependent translation, thereby leading to the selective reduction in TE of critical mRNA targets. Indeed, through an RNA-seq screen of polysomal RNA, a group of 122 mRNAs displaying reduced TE was identified in DAP5 knockdown hESCs, while global protein translation levels were not affected.

To identify direct DAP5 mRNA targets among these genes with reduced TE, candidates were screened for the ability of their $5^{\prime}$ UTRs to drive cap-independent translation. This was based on previous studies showing that DAP5 drives translation of its targets via IRES elements within the 5' UTR (Henis-Korenblit et al. 2002; Nevins et al. 2003; Warnakulasuriyarachchi et al. 2004; Lewis et al. 2008; Marash et al. 2008; Weingarten-Gabbay et al. 2014). We used a previously described function-based screen in a cell-free system (Liberman et al. 2015), since, despite some proposed in silico approaches (Baird et al. 2006; Wu et al. 2009; Weingarten-Gabbay et al. 2016), it is difficult to a priori predict IRESs within a candidate mRNA. In this manner, HMGN3 was identified to have cap-independent translation ability, which was further confirmed in vivo in hESCs by showing that its $5^{\prime}$ UTR drives DAP5-dependent/cap-independent translation. We showed previously that DAP5 interacts directly and specifically with its target p53 mRNA (Weingarten-Gabbay et al. 2014). Here we showed that the HMGN3 5' UTR can also interact with DAP5 in vivo by immunoprecipitation with DAP5 protein and in vitro by EMSA. Moreover, we mapped the interaction region to the first $\sim 100 \mathrm{nt}$ of the $5^{\prime}$ UTR. Most significantly, we found that HMGN3 function is critical for hESC differentiation, thereby linking DAP5-mediated translation of a specific target to its phenotype. Thus, HMGN3 provides the first proof of principle that selective translation of proteins through a cap-independent mechanism represents a new level of gene expression regulation controlling the transition from pluripotency to differentiation.

HMGN3 belongs to the HMGN family of chromatin architectural proteins. Each HMGN variant has specific biological functions differentially affecting the cellular transcriptional profile within specific tissues (Kugler et al. 2013). HMGN3 expression is high in adult brain and pancreatic islet cells, and HMGN3 knockout mice are viable but diabetic (Ito and Bustin 2002; Ueda et al. 2009; Kurahashi et al. 2010). Functionally, HMGN3 enhances transcription of its target genes by promoting histone acetylation and recruitment of transcription factors (Ueda et al. 2009; Barkess et al. 2012). Here we provide evidence for an additional role of HMGN3 during hESC differentiation. HMGN3 protein is expressed in pluripotent hESCs in a DAP5-dependent manner, and its knockdown resulted in changes in pluripotent and differentiation-associated gene expression upon RA treatment. Thus, HMGN3 joins other superfamily members, such as HMGN1, HMGN2, and HMGA, in regulating embryonic development (Furusawa and Cherukuri 2010; Ozturk et al. 2014).

Notably, out of seven mRNAs assayed in the in vitro translation screen, only HMGN3 showed significant cap-independent translation activity within its $5^{\prime}$ UTR. Considering previous reports showing that the $3^{\prime}$ UTR can also contribute to cap-independent translation (Izquierdo and Cuezva 2000; Martinez-Salas et al. 2008; Bradley et al. 2012; Bukhari et al. 2016; Weingarten-Gabbay et al. 2016), IRESs or elements that affect IRES activity may have been missed in this screen. Future screens can be conducted to include the $3^{\prime}$ UTR in the reporter plasmids and expand the number of targets assayed. Alternatively, the results may indicate that the identified genes with reduced TE include both direct targets of DAP5-mediated translation and indirect targets whose translation may depend on the former group. Indeed, the fact that the list was enriched in ribosomal proteins suggests that there may be a hierarchy of translation regulation, initiating with DAP5 and continuing with targets that regulate additional translation events. Further analysis of the translation cluster of potential DAP5 targets will enable investigation into this new direction in translation control of embryonic differentiation.

Another prominent find among the list of potential DAP5 mRNA targets was the mitochondria/oxidative phosphorylation annotation cluster, which was analyzed as a whole and shown to functionally contribute to the DAP5 knockdown phenotype. Pluripotent cells have been shown to be preferentially glycolytic, with a shift toward oxidative respiration occurring during the initial steps of ESC differentiation (Varum et al. 2011; Zhang et al. 2012). Moreover, the glycolytic intermediate acetyl-CoA is used for histone acetylation, an epigenetic mechanism for maintaining pluripotency (Moussaieff et al. 2015). Differentiation of stem cells is accompanied by enhanced mitochondrial biogenesis and oxidative respiration as well as elongation of mitochondria into a mature network, changes that occur even before loss of pluripotent markers (Mandal et al. 2011; Wanet et al. 2015). Significantly, we show here that inhibition of specific components of the oxidative phosphorylation pathway blocked RA-induced differentiation, consistent with similar inhibition studies during spontaneous differentiation of ESCs (Varum et al. 2009; Mandal et al. 2011; Zhang et al. 2011; Pereira et al. 2013), indicating that oxidative respiration is required for proper differentiation. Considering this, it is reasonable to conclude that the reduced translation of mitochondrial proteins, aberrant mitochondrial morphology, and decreased oxidative respiratory activity observed here upon DAP5 knockdown are contributing factors to the differentiation defect. Additional studies will address whether specific proteins within this cluster are direct IRES-dependent DAP5 targets.

Finally, it should be noted that the RNA-seq screen was conducted in pluripotent cells with the intention of 
identifying the earliest "priming" translation events common for differentiation toward different cell lineages. Future studies can assess whether additional DAP5 mRNA targets exist among mRNAs that are induced at later stages of cell lineage specification. For example, the phenotype of enhanced apoptosis in the growing EBs, but not after RA treatment, may result from reduced translation of anti-apoptotic genes expressed only during EB differentiation and not in the pluripotent state or other cell lineages.

In conclusion, for the first time, we linked DAP5 capindependent translation activity to regulation of hESC differentiation and exit from pluripotency. This function of DAP5 emphasizes the importance of noncanonical translation control for differentiation and provides a mechanistic explanation for the previously reported early embryonic lethality of DAP5 knockout mice and zebrafish (Yamanaka et al. 2000; Nousch et al. 2007). Furthermore, we showed the importance of the mitochondrial group of potential mRNA targets and a direct translation target, HMGN3, for hESC differentiation. This opens new prospects for identification of additional direct translation targets, especially among the mitochondria and ribosomal cohorts.

\section{Materials and methods}

Cell culture, EB formation, and generation of knockdown hESCs

hESC H9 (Wa-09) cells were maintained on a feeder layer of iMEFs (irradiated mouse embryonic fibroblasts) in hESC medium (DMEM F-12, 20\% knockout serum [ThermoFisher], 1\% NEAA, $0.5 \%$ glutamine, $0.1 \mathrm{mM} \beta$-mercaptoethanol, $8 \mathrm{ng} / \mathrm{mL}$ bFGF). The cells were grown for one passage before experiments on Matrigel-coated plates containing CM (conditioned medium). The medium was changed daily for hESCs grown in either condition. For differentiation, the medium was changed to differentiation medium (DMEM F-12, 20\% FBS, 1\% NEAA, 1\% glutamine, $1 \%$ ITSX), and all-trans RA (Sigma) was added at the indicated concentrations. Human EB (hEB) formation was induced from hESCs by gently passaging whole hESC colonies using $1 \mathrm{mg} / \mathrm{mL}$ collagenase IV. Detached cells were then plated on low-adhesion plates containing differentiation medium consisting of DMEM, $20 \%$ knockout serum, $1 \mathrm{mM}$ glutamine, $1 \%$ NEAA, and 0.1 $\mathrm{mM} \beta$-mercaptoethanol without bFGF. Cells were grown in these conditions for different periods of time (3-21 d), and the medium was changed every 2-3 d. Where indicated, DMSO or $10 \mu \mathrm{M}$ QVD-OPH (Biovision) was added to the EB medium at the time of plating. An additional $5 \mu \mathrm{M}$ Q-VD-OPH was added after $2 \mathrm{~d}$, and fresh medium supplemented with $10 \mu \mathrm{M}$ Q-VD-OPH was applied every $4 \mathrm{~d}$.

Stable knockdown of DAP5 was generated by infecting H9 hESCs with lentiviruses harboring pLKO.1-puro plasmid expressing shRNA to DAP5 (Sigma, TRCN0000147914) or NT followed by selection using puromycin. For transient transfections, including siRNA, H9 cells were plated on Matrigel-coated plates using StemPro Accutase (ThermoFisher). Twenty-four hours after plating, cells were transfected with $50 \mathrm{nmol}$ OnTargetPlus siRNA pool (Dharmacon) mixed with transfection reagent Dharmafect2 (Dharmacon). For rescue experiments, plasmids were transfected $24 \mathrm{~h}$ after siRNA transfection using Fugene6 (Promega) and changed to differentiation medium $24 \mathrm{~h}$ later.

\section{Spectral karyotyping (SKY)}

hESCs from DAP5 knockdown and NT knockdown were sent for karyotype testing using the SKY approach (Applied Spectral Imaging Ltd.). From each cell type, several slides were prepared and processed according to the company's SKY protocol.

\section{Histological analysis}

Cryo preservation hEBs were fixed in 4\% PFA and subjected to cryo-protection by a sucrose gradient $(10 \%-30 \%)$. The hEBs were then washed with PBS and moved to cryo-blocks. After drying, the blocks were frozen with optimal cutting temperature, and cryostat sections of $8 \mu \mathrm{m}$ were laid onto slides.

Paraffin-embedded sections hEBs were washed with PBS and fixed in $4 \%$ PFA for $30 \mathrm{~min}$. After washing, they were inserted into $3 \%$ agarose (low melting temperature) (Sigma), coated with paraffin, and sectioned to $8-10 \mu \mathrm{m}$ sections.

$H \& E$ staining H\&E staining was performed in the Weizmann Institute's Histology Unit using Tissue Stainer TST 40. The paraffin or cryo-sections were rehydrated by three successive washes with xylene followed by gradient washes with $100 \%$ to $70 \%$ ethanol and, finally, water. The slides were then stained with hematoxylin for $65 \mathrm{~min}$, washed with water and $70 \%$ ethanol, and then stained with eosin. After staining, slides were dehydrated with additional ethanol gradient washes and finally clarified by xylene washes. Slides were viewed and images were obtained with an Olympus BX41 microscope.

Immunostaining Fixed hESCs and hEB cryo-sections were washed in PBS. Permeabilization was performed with PBS + $0.2 \%$ Triton X-100 (PBT) followed by blocking in $0.1 \%$ PBT + $5 \%$ normal goat serum (NGS). Slides were incubated with primary antibody (see below for details of the antibodies used) overnight at $4^{\circ} \mathrm{C}$ followed by $1 \mathrm{~h}$ with the secondary antibody. Slides were then stained with DAPI and mounted with Immunomount. Slides were viewed and images were obtained with an Olympus BX41 microscope.

Mitotracker staining hESCs were grown on Matrigel-coated coverslips. The cells were incubated with $100 \mathrm{nM}$ MitoTracker Red CMXRos (ThermoFisher) in DMEM F-12 for 15 min at $37^{\circ} \mathrm{C}$ and washed with PBS. The cells were fixed with $4 \%$ PFA followed by PBS washes. The slides were incubated in ice-cold acetone for 5 min and washed again with PBS. Slides were then stained with DAPI and mounted with Immunomount. Slides were viewed with a Zeiss LSM780 confocal microscope system.

\section{Protein analysis}

Cells were lysed with RIPA buffer for protein purification, and Western blots were performed according to standard protocols. The following primary antibodies were used: OCT-4 (OCT3/4), Bcl-2, and ICAD (Santa Cruz Biotechnology, SC5279, SC-509, and SC-9066, respectively); Nanog and HMGN3 (Abcam, ab21624 and ab72233, respectively); REX-1 (R\&D, catalog no. MAB3598); GAPDH (Millipore, MAB374); PARP-1 (Biomol, SA250 and SA252); cleaved caspase-3 (Cell Signaling Technology, 9664); laminin (Sigma Aldrich, L9393); and Nat1/ DAP5 (Transduction Laboratories, N610020). Secondary antibodies were HRP-conjugated anti-mouse or anti-rabbit IgG (Jackson ImmunoResearch). 
For FACS analysis, hESCs and hEBs were grown to the relevant stages and dissociated using StemPro Accutase. Cells were resuspended in ice-cold PBS containing $0.05 \%$ sodium azide and $10 \%$ FBS with primary antibody to the cell surface protein TRA1-60 (Santa Cruz Biotechnology, SC-21705) and incubated for 1 h. Cells were then washed and stained with secondary antibody: Alexa fluor 555-conjugated goat anti-mouse IgG (ThermoFisher). Cells were then fixed, permeabilized, and stained with anticaspase-3 antibody (Cell Signaling Technology, 9664). After secondary antibody (anti-rabbit Cy5), the cells were washed, resuspended in PBS, and imaged using multispectral imaging flow cytometry (ImageStreamX flow cytometer, Amnis Corp.). Approximately $2 \times 10^{4}$ cells were collected from each sample, and data were analyzed using image analysis software (IDEAS 4.0, Amnis Corp.). Images were compensated for fluorescent dye overlap by using single-stain controls. Cells were gated for single cells using the area and aspect ratio features and for focused cells using the gradient RMS feature. Cells were gated for TRA1-60-positive and/or caspase3-positive cells according to their staining intensity.

\section{RNA analysis}

RNA was purified using RNeasy Plus minikit (Qiagen) or TRI reagent (Sigma). cDNA were reverse-transcribed with SuperScript II (Invitrogen), and real-time PCR was performed with Fast SYBR or TaqMan gene expression assays on StepOnePlus (Applied Biosystems, Life Technologies) according to the manufacturer's instructions. See Supplemental Table S2 for the primers used. Assays were performed in triplicates, normalized to RPLPO, and analyzed according to the $\Delta \Delta \mathrm{Ct}$ method. Data are representative of multiple experiments. TaqMan array human stem cell pluripotent panel (Applied Biosystems, Life Technologies) was used to screen stemness gene expression according to the manufacturer's protocol.

\section{Polysome fractionation and deep RNA-seq}

Polysomal fractionation of DAP5 and NT pluripotent hESCs, construction of RNA libraries from total poly $(\mathrm{A})^{+}$RNA and heavy polysomal fractions, and deep RNA-seq were performed as follows. Cyclohexamide $(\mathrm{CHX} ; 100 \mu \mathrm{g} / \mathrm{mL})$ was added to the medium of hESCs growing on Matrigel for $10 \mathrm{~min}$. Plates were washed with PBS + CHX and harvested in LBA $(20 \mathrm{mM}$ Tris at pH 7.5, 45 $\mathrm{mM} \mathrm{KCl}, 10 \mathrm{mM} \mathrm{MgCl}_{2}, 0.1 \mathrm{mg} / \mathrm{mL}$ CHX, $1 \mathrm{mMDTT}, 2.5 \mu \mathrm{L} / \mathrm{mL}$ RNasin, protease inhibitor cocktail) with $1 \%$ NP-40. Cleared lysate ( 12 OD) was loaded onto $10 \%-50 \%$ sucrose gradients in LBA and centrifuged at $39,000 \mathrm{rpm}$ for $100 \mathrm{~min}$ in a SW-41 rotor (Optima LE-80k Ultracentrifuge, Beckman). The gradients were collected with Teledyne ISCO (UA-6), and $0.5 \mathrm{~mL}$ fractions were collected. Thirty microliters of $10 \%$ SDS and $1 \mathrm{~mL}$ of $100 \%$ ethanol were added to each fraction. The fractions were incubated overnight at $-20^{\circ} \mathrm{C}$ and centrifuged at 20,000 g for $15 \mathrm{~min}$. The pellet was resuspended in TRI reagent, and RNA was purified according to the protocol. For total RNA, the same procedure was done directly on lysate samples.

Fractions 19-23, consisting of heavy polysomes, and total RNA $(0.5 \mu \mathrm{g})$ were processed using the TruSeq RNA sample preparation kit protocol (Illumina) according to the manufacturer's instructions. Libraries were evaluated by Qubit and Bioanalyzer. Sequencing libraries were constructed with barcodes to allow multiplexing of eight samples on one lane. The sequencing was run on an Illumina HiSeq 2000 instrument. The obtained reads, paired-ended 100 base pairs (bp) long, were mapped using TopHat version 1.3.3 against the human genome (hg19 downloaded from University of California at Santa Cruz).
Expression at the gene level was quantified by applying the Partek Genomics suite and using RefSeq in gtf format as an annotation. Differential expression was calculated using DESeq2 software (version 1.2.8) (Love et al. 2014). The cutoff for differentially expressed genes was at least 20 reads, an absolute fold change of 2, and false discovery rate (FDR) of 0.05 . For analysis of translational changes, a two-factor model-including the RNA type (total or polysomal) and the genotype (NT or DAP5 knockdown) and the interaction between them-was built using DESeq2. Specifically, for each genotype, the counts within the polysomal fraction were divided by the counts within the total mRNA to obtain the TE $\left(\mathrm{TE}_{\text {shDAP5 }}=\right.$ Poly $_{\text {shDAP5 }} /$ Total $_{\text {shDAP5 }}$ or $\mathrm{TE}_{\text {shNT }}=$ Poly $_{\text {shNT }} /$ Total $\left._{\text {shNT }}\right) . \mathrm{TE}_{\text {shDAP5 }}$ was then divided into $\mathrm{TE}_{\text {shNT }}$ to obtain the differential TE ( $\left.\triangle \mathrm{TE}\right)$. Genes with $\triangle \mathrm{TE}$ values less than -1.5 (with an FDR threshold of at least 0.05) were considered to have significantly changed. RNA-seq data sets were deposited in Gene Expression Omnibus with accession number GSE80467.

\section{In vitro and in vivo IRES activity assays}

$B C L 2$ IRES and empty FL vectors were previously described (Liberman et al. 2015). The 5' UTRs of the seven candidate genes were cloned by PCR amplification from $\mathrm{H} 9$ total cDNA or synthesized plasmid expression vectors (Genewiz) and inserted into the FL reporter plasmid. The genes and primers used for cloning are in Supplemental Table S3. RNA transcripts were produced using the RiboMax kit as described by the manufacturer (Promega). m7GpppG (m7G cap) (New England BioLabs) was added to the reaction at $10 \mathrm{mM}$ concentration. Poly(A) tailing was not required, as the FL constructs contained a poly(A) sequence of $35 \mathrm{bp}$. Final recovery of RNA transcripts was achieved with the RNeasy MinElute cleanup kit (Qiagen).

For in vitro translation assays, RNA was added to RRLs $160 \%-$ $75 \%[\mathrm{v} / \mathrm{v}]$ ), treated with micrococcal nuclease (Promega), and supplemented with $0.01 \mathrm{mM}$ mixture of amino acids and $0.8 \mathrm{U} / \mathrm{\mu L}$ RNasin (Promega). Reaction samples were incubated for $1 \mathrm{~h}$ at $30^{\circ} \mathrm{C}$. Luciferase activity was measured using the luciferase assay system and a Veritas microplate luminometer (Promega) as described by the manufacturer. For in vivo IRES activity, the plasmids were transfected into hESCs using Fugene6. Forty-eight hours later, cells were lysed with passive lysis buffer (PLB) (Promega), and luciferase activity was measured. Luciferase activity was normalized to luciferase mRNA expression measured by quantitative RT-PCR, as described above.

\section{RNA immunoprecipitation}

H9 cells grown on Matrigel were UV-cross-linked at $0.15 \mathrm{~mJ} / \mathrm{cm}^{2}$ (254 nm). Cells were harvested and snap-frozen. The cell pellets were resuspended in lysis buffer $(50 \mathrm{mM}$ Tris- $\mathrm{HCl}$ at $\mathrm{pH} 7.4$, $100 \mathrm{mM} \mathrm{NaCl}, 1 \% \mathrm{NP}-40,0.1 \%$ SDS, $0.5 \%$ sodium deoxycholate, with protease inhibitors), and the DAP5-RNA complexes were immunoprecipitated with rabbit anti-DAP5 monoclonal antibody (Cell Signaling, 5169) followed by stringent washes with up to $1 \mathrm{M} \mathrm{NaCl}$. The bound RNA was extracted with TRI reagent, cDNA was generated, and quantitative RT-PCR was performed as described. RNA immunoprecipitation with anti-DAP5 antibody was normalized to the corresponding levels within total mRNA (input RNA).

\section{RNA-binding assay (EMSA)}

$\left[{ }^{32} \mathrm{P}\right]$ UTP-labeled HMGN3 $5^{\prime}$ UTR nucleotides -178 to +30 or each of its two halves $(10,000$ counts per minute) was incubated 
for $20 \mathrm{~min}$ at $30^{\circ} \mathrm{C}$ with different amounts (300 or $600 \mathrm{ng}$ ) of recombinant DAP5 protein (Liberman et al. 2015) in $15 \mu \mathrm{L}$ of buffer $\mathrm{B}(20 \mathrm{mM}$ Tris- $\mathrm{HCl}$ at $\mathrm{pH} 7.5,100 \mathrm{mM} \mathrm{KCl}, 4 \mathrm{mM}$ DTT, $0.01 \%$ NP-40, $2 \mathrm{mM} \mathrm{MgAc}$. For the competition assays, calculated molar excess of unlabeled RNAs was added along with the components of the reaction mixture and $600 \mathrm{ng}$ of DAP5 protein. Sample buffer was added to the reaction mixtures prior to gel electrophoresis on a $6 \%$ polyacrylamide gel.

\section{Respiratory chain enzymatic activities}

The mitochondrial-enriched fraction was isolated from cells by homogenization on ice in isolation buffer $(320 \mathrm{mM}$ sucrose, 5 $\mathrm{mM}$ Tris- $\mathrm{HCl}$ at $\mathrm{pH} 7.4,2$ mM EGTA). The homogenate was centrifuged at $2000 \mathrm{~g}$ for $3 \mathrm{~min}$ at $4^{\circ} \mathrm{C}$ to remove nuclei, and the resulting supernatant was centrifuged at $12,000 \mathrm{~g}$ for $10 \mathrm{~min}$ at $4^{\circ} \mathrm{C}$ to pellet mitochondria, which were stored in isolation buffer at $-70^{\circ} \mathrm{C}$ until use. The enzymatic activity of respiratory chain complex I (rotenone-sensitive NADH-CoQ reductase) was measured as reported previously (Shufaro et al. 2012) by monitoring the oxidation of NADH by spectrophotometry at $340 \mathrm{~nm}$ at $37^{\circ}$ $\mathrm{C}$ in the presence of coenzyme $\mathrm{Q}_{1}$. Activity of the mitochondrial matrix enzyme citrate synthase, used as a control, was measured in the presence of acetyl-CoA and oxaloacetate by monitoring the release of CoASH coupled to $5^{\prime}, 5^{\prime}$-dithiobis (2-nitrobenzoic) acid at $412 \mathrm{~nm}$. Activities are reported as the ratio of complex I to citrate synthase $(\mathrm{U} / \mathrm{U})$.

\section{Acknowledgments}

We acknowledge N. Benvenisty for critical reading of the manuscript, J.H. Hanna for helpful discussions, and Corinne Alban for technical assistance. This project was funded by the Israel Science Foundation (grant no. 133/12) and the Kimmel Center for Stem Cell Research. A.K. is the Incumbent of the Helena Rubinstein Chair of Cancer Research. G.F. is the Incumbent of the David and Stacey Cynamon Research Fellow Chair in Genetics and Personalized Medicine.

\section{References}

Baird SD, Turcotte M, Korneluk RG, Holcik M. 2006. Searching for IRES. RNA 12: 1755-1785.

Barkess G, Postnikov Y, Campos CD, Mishra S, Mohan G, Verma S, Bustin M, West KL. 2012. The chromatin-binding protein HMGN3 stimulates histone acetylation and transcription across the Glytl gene. Biochem I 442: 495-505.

Bradley S, Narayanan S, Rosbash M. 2012. NAT1/DAP5/p97 and atypical translational control in the Drosophila Circadian Oscillator. Genetics 192: 943-957.

Bukhari SI, Truesdell SS, Lee S, Kollu S, Classon A, Boukhali M, Jain E, Mortensen RD, Yanagiya A, Sadreyev RI, et al. 2016. A specialized mechanism of translation mediated by FXR1a-associated microRNP in cellular quiescence. Mol Cell 61: 760-773.

Caserta TM, Smith AN, Gultice AD, Reedy MA, Brown TL. 2003. Q-VD-OPh, a broad spectrum caspase inhibitor with potent antiapoptotic properties. Apoptosis 8: 345-352.

Coucouvanis E, Martin GR. 1995. Signals for death and survival: a two-step mechanism for cavitation in the vertebrate embryo. Cell 83: 279-287.

Coucouvanis E, Martin GR. 1999. BMP signaling plays a role in visceral endoderm differentiation and cavitation in the early mouse embryo. Development 126: 535-546.
Dhara SK, Stice SL. 2008. Neural differentiation of human embryonic stem cells. J Cell Biochem 105: 633-640.

Easley CA IV, Ben-Yehudah A, Redinger CJ, Oliver SL, Varum ST, Eisinger VM, Carlisle DL, Donovan PJ, Schatten GP. 2010. mTOR-mediated activation of p70 S6K induces differentiation of pluripotent human embryonic stem cells. Cell Reprogram 12: 263-273.

Furusawa T, Cherukuri S. 2010. Developmental function of HMGN proteins. Biochim Biophys Acta 1799: 69-73.

Henis-Korenblit S, Strumpf NL, Goldstaub D, Kimchi A. 2000. A novel form of DAP5 protein accumulates in apoptotic cells as a result of caspase cleavage and internal ribosome entry sitemediated translation. Mol Cell Biol 20: 496-506.

Henis-Korenblit S, Shani G, Sines T, Marash L, Shohat G, Kimchi A. 2002. The caspase-cleaved DAP5 protein supports internal ribosome entry site-mediated translation of death proteins. Proc Natl Acad Sci 99: 5400-5405.

Huang DW, Sherman BT, Lempicki RA. 2009a. Bioinformatics enrichment tools: paths toward the comprehensive functional analysis of large gene lists. Nucleic Acids Res 37: $1-13$.

Huang DW, Sherman BT, Lempicki RA. 2009b. Systematic and integrative analysis of large gene lists using DAVID bioinformatics resources. Nat Protoc 4: 44-57.

Imataka H, Olsen HS, Sonenberg N. 1997. A new translational regulator with homology to eukaryotic translation initiation factor 4G. $E M B O T$ 16: 817-825.

Ito Y, Bustin M. 2002. Immunohistochemical localization of the nucleosome-binding protein HMGN3 in mouse brain. J Histochem Cytochem 50: 1273-1275.

Izquierdo JM, Cuezva JM. 2000. Internal-ribosome-entry-site functional activity of the $3^{\prime}$-untranslated region of the mRNA for the $\beta$ subunit of mitochondrial $\mathrm{H}^{+}$-ATP synthase. Biochem I 346(Pt 3): 849-855.

Kugler JE, Horsch M, Huang D, Furusawa T, Rochman M, Garrett L, Becker L, Bohla A, Holter SM, Prehn C, et al. 2013. High mobility group $\mathrm{N}$ proteins modulate the fidelity of the cellular transcriptional profile in a tissue- and variant-specific manner. J Biol Chem 288: 16690-16703.

Kurahashi T, Furusawa T, Ueda T, Bustin M. 2010. The nucleosome binding protein HMGN3 is expressed in pancreatic acells and affects plasma glucagon levels in mice. I Cell Biochem 109: 49-57.

Levy-Strumpf N, Deiss LP, Berissi H, Kimchi A. 1997. DAP-5, a novel homolog of eukaryotic translation initiation factor 4G isolated as a putative modulator of $\gamma$ interferon-induced programmed cell death. Mol Cell Biol 17: 1615-1625.

Lewis SM, Cerquozzi S, Graber TE, Ungureanu NH, Andrews M, Holcik M. 2008. The eIF4G homolog DAP5/p97 supports the translation of select mRNAs during endoplasmic reticulum stress. Nucleic Acids Res 36: 168-178.

Liberman N, Gandin V, Svitkin YV, David M, Virgili G, Jaramillo M, Holcik M, Nagar B, Kimchi A, Sonenberg N. 2015. DAP5 associates with eIF2 $\beta$ and eIF4AI to promote internal ribosome entry site driven translation. Nucleic Acids Res 43: 3764-3775.

Love MI, Huber W, Anders S. 2014. Moderated estimation of fold change and dispersion for RNA-seq data with DESeq2. Genome Biol 15: 550.

Mandal S, Lindgren AG, Srivastava AS, Clark AT, Banerjee U. 2011. Mitochondrial function controls proliferation and early differentiation potential of embryonic stem cells. Stem Cells 29: 486-495.

Marash L, Liberman N, Henis-Korenblit S, Sivan G, Reem E, Elroy-Stein O, Kimchi A. 2008. DAP5 promotes cap- 
independent translation of Bcl-2 and CDK1 to facilitate cell survival during mitosis. Mol Cell 30: 447-459.

Martinez-Salas E, Pacheco A, Serrano P, Fernandez N. 2008. New insights into internal ribosome entry site elements relevant for viral gene expression. J Gen Virol 89: 611-626.

Moussaieff A, Rouleau M, Kitsberg D, Cohen M, Levy G, Barasch D, Nemirovski A, Shen-Orr S, Laevsky I, Amit M, et al. 2015. Glycolysis-mediated changes in acetyl-CoA and histone acetylation control the early differentiation of embryonic stem cells. Cell Metab 21: 392-402.

Nevins TA, Harder ZM, Korneluk RG, Holcik M. 2003. Distinct regulation of internal ribosome entry site-mediated translation following cellular stress is mediated by apoptotic fragments of eIF4G translation initiation factor family members eIF4GI and p97/DAP5/NAT1. J Biol Chem 278: 35723579.

Nousch M, Reed V, Bryson-Richardson RJ, Currie PD, Preiss T. 2007. The eIF4G-homolog p97 can activate translation independent of caspase cleavage. RNA 13: 374-384.

Novak A, Amit M, Ziv T, Segev H, Fishman B, Admon A, Itskovitz-Eldor J. 2012. Proteomics profiling of human embryonic stem cells in the early differentiation stage. Stem Cell Rev 8: 137-149.

Orkin SH, Hochedlinger K. 2011. Chromatin connections to pluripotency and cellular reprogramming. Cell 145: 835-850.

Ozturk N, Singh I, Mehta A, Braun T, Barreto G. 2014. HMGA proteins as modulators of chromatin structure during transcriptional activation. Front Cell Dev Biol 2: 5.

Pereira SL, Graos M, Rodrigues AS, Anjo SI, Carvalho RA, Oliveira PJ, Arenas E, Ramalho-Santos J. 2013. Inhibition of mitochondrial complex III blocks neuronal differentiation and maintains embryonic stem cell pluripotency. PLoS One 8: e82095.

Qi Y, Tian X, Liu J, Han Y, Graham AM, Simon MC, Penninger JM, Carmeliet P, Li S. 2012. Bnip3 and AIF cooperate to induce apoptosis and cavitation during epithelial morphogenesis. $J$ Cell Biol 198: 103-114.

Sampath P, Pritchard DK, Pabon L, Reinecke H, Schwartz SM, Morris DR, Murry CE. 2008. A hierarchical network controls protein translation during murine embryonic stem cell self-renewal and differentiation. Cell Stem Cell 2: 448-460.

Shaughnessy JD Jr, Jenkins NA, Copeland NG. 1997. cDNA cloning, expression analysis, and chromosomal localization of a gene with high homology to wheat eIF-(iso)4F and mammalian eIF-4G. Genomics 39: 192-197.

Shufaro Y, Lebovich M, Aizenman E, Miller C, Simon A, Laufer N, Saada A. 2012. Human granulosa luteal cell oxidative phosphorylation function is not affected by age or ovarian response. Fertil Steril 98: 166-172.

Tahmasebi S, Alain T, Rajasekhar VK, Zhang JP, Prager-Khoutorsky M, Khoutorsky A, Dogan Y, Gkogkas CG, Petroulakis E, Sylvestre A, et al. 2014. Multifaceted regulation of somatic cell reprogramming by mRNA translational control. Cell Stem Cell 14: 606-616.

Thomson JA, Itskovitz-Eldor J, Shapiro SS, Waknitz MA, Swiergiel JJ, Marshall VS, Jones JM. 1998. Embryonic stem cell lines derived from human blastocysts. Science 282: 11451147.

Ueda T, Furusawa T, Kurahashi T, Tessarollo L, Bustin M. 2009. The nucleosome binding protein HMGN3 modulates the transcription profile of pancreatic $\beta$ cells and affects insulin secretion. Mol Cell Biol 29: 5264-5276.

Varum S, Momcilovic O, Castro C, Ben-Yehudah A, RamalhoSantos J, Navara CS. 2009. Enhancement of human embryonic stem cell pluripotency through inhibition of the mitochondrial respiratory chain. Stem Cell Res 3: 142-156.

Varum S, Rodrigues AS, Moura MB, Momcilovic O, Easley CA IV, Ramalho-Santos J, Van Houten B, Schatten G. 2011. Energy metabolism in human pluripotent stem cells and their differentiated counterparts. PLoS One 6: e20914.

Wanet A, Arnould T, Najimi M, Renard P. 2015. Connecting mitochondria, metabolism, and stem cell fate. Stem Cells Dev 24: 1957-1971.

Warnakulasuriyarachchi D, Cerquozzi S, Cheung HH, Holcik M. 2004. Translational induction of the inhibitor of apoptosis protein HIAP2 during endoplasmic reticulum stress attenuates cell death and is mediated via an inducible internal ribosome entry site element. J Biol Chem 279: 17148-17157.

Weingarten-Gabbay S, Khan D, Liberman N, Yoffe Y, Bialik S, Das S, Oren M, Kimchi A. 2014. The translation initiation factor DAP5 promotes IRES-driven translation of p53 mRNA. Oncogene 33: 611-618.

Weingarten-Gabbay S, Elias-Kirma S, Nir R, Gritsenko AA, Stern-Ginossar N, Yakhini Z, Weinberger A, Segal E. 2016. Comparative genetics. Systematic discovery of cap-independent translation sequences in human and viral genomes. Science 351.

Wu TY, Hsieh CC, Hong JJ, Chen CY, Tsai YS. 2009. IRSS: a Webbased tool for automatic layout and analysis of IRES secondary structure prediction and searching system in silico. BMC BiOinformatics 10: 160.

Yamanaka S, Poksay KS, Arnold KS, Innerarity TL. 1997. A novel translational repressor mRNA is edited extensively in livers containing tumors caused by the transgene expression of the apoB mRNA-editing enzyme. Genes Dev 11:321-333.

Yamanaka S, Zhang XY, Maeda M, Miura K, Wang S, Farese RV Jr, Iwao H, Innerarity TL. 2000. Essential role of NAT1/p97/ DAP5 in embryonic differentiation and the retinoic acid pathway. EMBO J 19: 5533-5541.

Yeo JC, Ng HH. 2013. The transcriptional regulation of pluripotency. Cell Res 23: 20-32.

Young RA. 2011. Control of the embryonic stem cell state. Cell 144: 940-954.

Zhang J, Khvorostov I, Hong IS, Oktay Y, Vergnes L, Nuebel E, Wahjudi PN, Setoguchi K, Wang G, Do A, et al. 2011. UCP2 regulates energy metabolism and differentiation potential of human pluripotent stem cells. EMBO $I$ 30: 4860-4873.

Zhang J, Nuebel E, Wisidagama DR, Setoguchi K, Hong JS, Van Horn CM, Imam SS, Vergnes L, Malone CS, Koehler CM, et al. 2012. Measuring energy metabolism in cultured cells, including human pluripotent stem cells and differentiated cells. Nat Protoc 7: 1068-1085. 


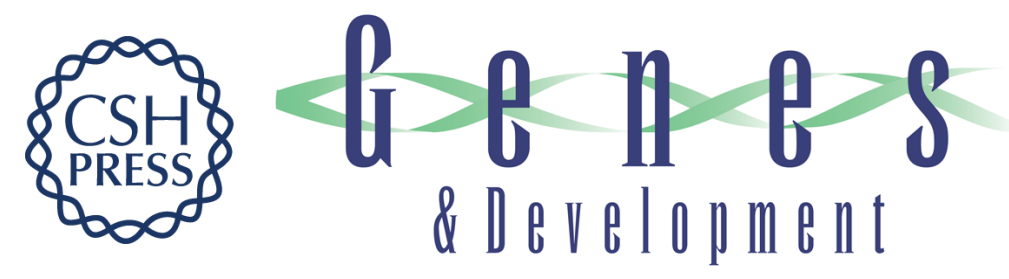

\section{Cap-independent translation by DAP5 controls cell fate decisions in human embryonic stem cells}

Yael Yoffe, Maya David, Rinat Kalaora, et al.

Genes Dev. 2016, 30:

Access the most recent version at doi:10.1101/gad.285239.116

\section{Supplemental http://genesdev.cshlp.org/content/suppl/2016/09/23/30.17.1991.DC1 Material}

References This article cites 52 articles, 19 of which can be accessed free at: http://genesdev.cshlp.org/content/30/17/1991.full.html\#ref-list-1

Creative This article, published in Genes \& Development, is available under a Creative Commons Commons License (Attribution-NonCommercial 4.0 International), as described at License http://creativecommons.org/licenses/by-nc/4.0/.

Email Alerting Receive free email alerts when new articles cite this article - sign up in the box at the top Service right corner of the article or click here.

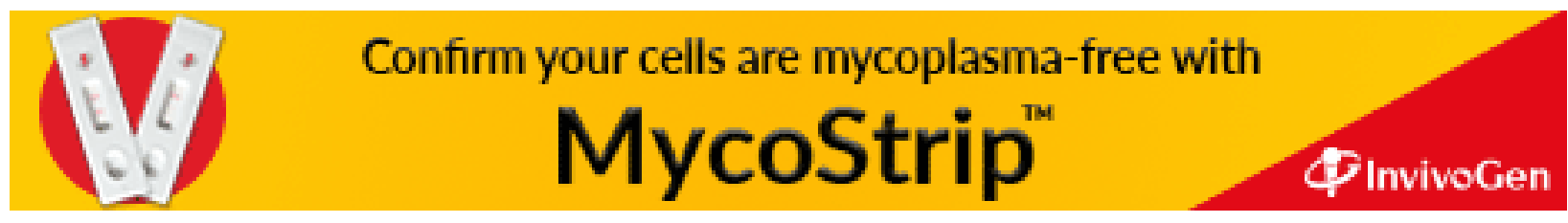

